rich plasma (PRP) have been ascribed to its ability to rectify intra-articular inflammatory processes.

Objectives: The aim of this study was to determine the safety, feasibility and changes in symptom severity, sports activity, lower limb function, and function in daily living activities following intra-articular injections of PRP in mild to moderate knee OA.

Methods: In this double-blind randomized controlled pilot study, 34 patients with knee OA grade 2 or 3, from March 2013 to February 2016 were selected. Participants were randomly assigned one knee as the case and other knee was considered as control to receive three injections of either PRP or placebo. The administering doctor and the patients were blinded to group allocation. Outcomes included safety and recruitment data, $100 \mathrm{~mm}$ the EuroQol-visual analogue scales (EQ-VAS), the international knee documentation committee (IKDC), the Western Ontario and McMaster Universities Arthritis Index (WOMAC) and the Tegner Activity Score (TAS) at three and six months.

Results: Twenty four (100\%) participants met the inclusion criteria. No treatmentrelated major adverse events were reported. The PRP group demonstrated significant improvements at all follow up time points in the WOMAC ( 3 months: $p=0.017 ; 6$ months: $p=0.029$, $E T A=0.601$ ). For the PRP group, the $11.47 \mathrm{~mm}$ reduction in EQ-VAS at 6 months $(p=0.040)$ and the $7.35 \mathrm{~mm}$ at 3 months $(\mathrm{p}=0.035)$ was statistically significant improvement from baseline. The PRP group also significantly improved IKDC $(p=0.039$, ETA $=0.619)$ and TAS $(p=0.028$, $E T A=0.641$ ) at three months. The placebo group showed improvements on only the IKDC Function at 3 months $(p=0.019$, ETA $=0.591)$. There were no significant between-group differences for any of the self-reported measures at either time-point.

Conclusions: The study provides proof-of-concept evidence about the safety and feasibility of intra-articular injections of PRP necessary to appraise a larger clinical trial knee OA. Our preliminary findings also suggest PRP improves self-reported symptom severity, sports activity, and function in daily living activities, however no between-group differences were found. PRP may provide an effective and safe novel treatment for knee OA.

Acknowledgements: This study was supported by Semnan University of Medical Sciences, Semnan, Iran.

Disclosure of Interest: None declared

DOI: 10.1136/annrheumdis-2017-eular.1387

\section{AB0804 SINGLE-NUCLEOTIDE POLYMORPHISM (SNP) RS143383 GROWTH AND DIFFERENTIATION FACTOR 5 (GDF5) IN KNEE OSTEOARTHRITIS IN EGYPTIAN POPULATION}

M. Hassanien ${ }^{1}$, N.A. Mohamed ${ }^{2}$, S.A. Mahran ${ }^{1} .{ }^{1}$ Rheumatology Department; ${ }^{2}$ Medical Biochemistry, Assuit University, assuit, Egypt

Background: Osteoarthritis $(\mathrm{OA})$, is an age-related common polygenic disease characterized by the thinning and loss of the articular cartilage in synovial joints such as knees. The etiology and pathogenesis of $\mathrm{OA}$ is largely unknown, the singlenucleotide polymorphism (SNP) rs143383 (C/T) influencing OA susceptibility across a range of ethnic groups.

Objectives: The present study investigated to identify the association of polymorphism in GDF-5 gene with osteoarthritis in Egyptian population.

Methods: This Case control study of 100 women and 100 male $\geq 40$ years that fulfilled American College of Rheumatology (ACR) for Knee OA and 100 controls recruited from the outpatient clinic of Department of Rheumatology, Asyut university, Egypt. Clinical symptoms were assessed with WOMAC index and VAS for knee pain. The severity of disease was determined by radiological grades (Kellgren Lawren). Body Mass Index (BMI) was recorded. DNA isolation and genotype analysis the method of (Southam et al., 2007) was followed for determining the GDF-5 gene (T/C; rs143383) polymorphism. Amplification was performed.

Results: There were weak but significant associations present between the GDF5 polymorphism and knee OA at the allele level (C vs. T: $0.85,95 \% \mathrm{Cl}=0.78-0.93$ ) and genotype level (CC vs. TT: 0.72 ; CT vs. TT: $0.81 ; \mathrm{CC} / \mathrm{CT}$ vs. TT: 0.83 ; CC vs. CT/TT: 0.78 in the overall population. A stronger significant association was observed for $\mathrm{CC}$ vs. TT $(\mathrm{OR}=0.83, \mathrm{P}<0.001)$ in comparison with other models. In males we identified a second polymorphism, located in the 3'-UTR of GDF5, that influenced allelic expression of the gene independent of rs 143383 .

Conclusions: GDF5 is an OA susceptibility gene with association between the GDF5 polymorphism and clinical symptoms of knee OA in Egyptian population.

Disclosure of Interest: None declared

DOI: 10.1136/annrheumdis-2017-eular.1273

\section{AB0805 PANLAR CONSENSUS. RECOMMENDATIONS FOR THE MANAGEMENT OF OSTEOARTHRITIS OF THE HAND, HIP AND KNEE. SHORT TITLE: PAN-AMERICAN LEAGUE OF ASSOCIATIONS FOR RHEUMATOLOGY (PANLAR) OSTEOARTHRITIS (OA) STUDY GROUP}

M.J. Quintero ${ }^{1}$, H. Riera ${ }^{2}$, R. Espinosa ${ }^{3}$, R. Souto ${ }^{4}$, C. Rossi ${ }^{5}$, J.F. Molina ${ }^{6}$, J. Salas ${ }^{7}$, F. Radrigan ${ }^{8}$, G. Reyes ${ }^{9}$, L. Urioste ${ }^{10}$, S. Papasidero ${ }^{11}$, A. Garcia ${ }^{12}$ I. Iraheta ${ }^{13}$, E. Nieto ${ }^{2}$, R. Arape $^{14}$, O. Castaneda $^{15}$, J. Angulo ${ }^{16}$, I. Coimbra ${ }^{17}$, L.M. Franco ${ }^{18}$, C. Acosta ${ }^{19}$, V. Liendo ${ }^{20}$, R. Chacón-Díaz ${ }^{2}$, M. Vera ${ }^{2}$,
A. Reginato ${ }^{21}$, C.V. Caballero ${ }^{22}$, O. Rillo ${ }^{23}$ on behalf of (PANLAR) Osteoarthritis (OA) Study Group. ${ }^{1}$ ReumatologíA; ${ }^{2}$ Universidad de Los Andes, Mérida, Venezuela, Bolivarian Reoublic of, Mérida, Venezuela, Bolivarian Republic Of; ${ }^{3}$ Reumatología, Instituto Nacional de Rehabilitación, Ciudad de México, Mexico; ${ }^{4}$ Cátedra de Reumatología de la Facultad de Medicina de la Universidad de la Republica; ${ }^{5}$ Cátedra de Reumatología de la Facultad de Medicina de la Universidad de la Republica, Montevideo, Uruguay; ${ }^{6}$ Rheumatology and the Arthritis Clinical Research Unit at Medicarte, Medellin; ${ }^{7}$ Grupo de Reumatólogos del Caribe, Barranquilla, Colombia; ${ }^{8}$ Universidad Católica de Chile, Santiago, Chile; ${ }^{9}$ Servicio de Reumatología, Hospital Quirúrgico 10 de Octubre, Cerro, la Habana, Cuba; ${ }^{10}$ Universidad Católica San Pablo, Bolivia, Bolivia, Plurinational State Of: ${ }^{11}$ Servicio de Reumatología del Hospital General de Agudos Dr. E. Tornú, Buenos Aires, Argentina; ${ }^{12}$ Facultad de Medicina, Universidad Francisco Marroquín; ${ }^{13}$ Facultad de Medicina, Universidad Francisco Marroquín, Ciudad de Guatemala, Guatemala; ${ }^{14}$ Centro Clínico la Isabelica, Carabobo, Venezuela, Bolivarian Republic Of; ${ }^{15}$ Universidad Peruana de Ciencias Aplicadas;

${ }^{16}$ Universidad Nacional Mayor de San Marcos, Peru, Peru; ${ }^{17}$ Universidade Estadual de Campinas, Brasil, Brazil; ${ }^{18}$ Instituto de Urología, Valencia;

${ }^{19}$ Complejo Hospitalario Universitario "Ruiz y Páez", Ciudad Bolívar; ${ }^{20}$ Unidad de investigación Reumatológica. Clínica Félix Boada., Caracas, Venezuela,

Bolivarian Republic Of; ${ }^{21}$ División of Rheumatology, The Warren Alpert School of Medecine., Providence, United States; ${ }^{22}$ Universidad de la Sabana, Barranquilla, Colombia; ${ }^{23}$ Hospital Pirovano, Buenos Aires, Argentina

Background: This consensus derives from a previous study of Demographic and Clinical Characteristics by the PANLAR OA study group, reporting significant differences in handling these patients and in which it was evident the need of reaching an agreement in the management of hand, hip and knee OA in Latin America (2)

Objectives: The aim is to update the recommendations for the treatment of hand, hip and knee OA by agreeing on key propositions relating to the management of hand, hip and knee OA,identifying and critically appraising research evidence for the effectiveness of the treatments and by generating recommendations based on the available evidence and expert opinion.

Methods: Recommendations were developed by a group of 40 specialists made up of rheumatologists and members of other medical disciplines. A systematic review of articles, meta-analyses and guidelines for the management of hand, hip and knee OA published from 2008 and January 2014 was done. The level of evidence and strength of recommendation were classified according to the Jadad scale (3). The level of agreement was established through a Delphi technique.

Results: Both "strong" and "conditional" recommendations are given for management of hand, hip and knee OA and non-pharmacological, pharmacological and surgical modalities of treatment are presented according to the different levels of agreement.

Conclusions: These recommendations are based on the consensus of clinical experts from a wide range of disciplines considering the available evidence, while balancing the benefits and risks of non-pharmacological, pharmacological and surgical treatment modalities. It is hoped that these recommendations will be utilized by healthcare providers involved in the management of patients with hand, hip and knee OA.

References:

[1] Reginato, Anthony M. et al. Osteoarthritis in Latin America: Study of Demographic and Clinical Characteristics in 3040 Patients. JCR: Journal of Clinical Rheumatology 21.8(2015):391-397.

[2] Jadad, A.R.; Moore R.A.; Carroll D.; Jenkinson C.; Reynolds D.J.M.; Gavaghan D.J.; McQuay H.J.(1996). Assessing the quality of reports of randomized clinical trials: Is blinding necessary? Controlled Clinical Trials 17:1-12. doi: 10.1016/ 0197-2456(95)00134-4. PMID 8721797.

Disclosure of Interest: None declared

DOI: 10.1136/annrheumdis-2017-eular.6001

\section{AB0806 THE EFFECT OF REPETITIVE ACTIVE RANGE OF MOTION VERSUS CONTINUOUS PASSIVE MOTION ON EARLY FUNCTIONAL OUTCOMES AFTER PRIMARY TOTAL KNEE REPLACEMENT}

M. Erduran ${ }^{1}$, M. Eymir ${ }^{2}$, B. Ünver ${ }^{2}{ }^{1}$ Faculty of Medicine Surgery Medicine Department of Orthopaedics and Traumatology, Dokuz Eylül University, Izmir, Turkey; ${ }^{2}$ School of Physical Therapy and Rehabilitation, Dokuz Eylül University, Izmir, Turkey, Izmir, Turkey

Background: Continuous passive motion (CPM) is a common procedure in total knee replacement (TKR), but its effectiveness is controversial at early stage after TKR. Some studies have claimed that CPM promoting rapid postoperative recovery and the range of motion (ROM). However, some studies have demonstrated that CPM have any benefit on ROM, length stay of hospital and postoperative recovery $(1,2)$. Several studies have examined the use of CPM, but there is not any study that compared the effect of repetitive active range of motion (AROM) vs. CPM on early functional outcomes after TKR.

Objectives: The aim of this study was to compare the effect of AROM vs. CPM on early functional outcomes after TKR.

Methods: The study group consisted of 71 patients, who underwent primary TKR because of arthrosis were consecutively allocated to a AROM group $(n=40$, with 
median age; $70.8 \pm 7.9$ years), and were allocated to a CPM group ( $\mathrm{n}=31$, with median age; $69.7 \pm 8.4$ years). Patients were evaluated regarding knee function score (Hospital for Special Surgery (HSS) score), pain (Numeric Pain Rating Scale (NPRS)), knee circumference, knee range of motion, length of hospital stay, the day of knee flexion angle achieved 70 degrees and quality of life (Short-Form 12 Health Survey (SF-12). Functional activities were evaluated using the lowa Level of Assistance Scale and walking speed was evaluated using the lowa Ambulation Velocity Scale. Also functional outcomes were evaluated with timed up and go (TUG) test and 10-meter walk test (10 MWT). Patients were evaluated preoperatively and at discharge.

Results: At baseline, demographic and anthropometric characteristics were similar in groups and there was no statistically difference between groups $(p>0.05)$. It was determined that; the AROM group had better results in terms of reduction of pain severity $(p<0.001)$. When the HSS knee scores were compared there was statistically difference between groups $(p<0.001)$ and the HSS knee scores were lower in CPM group after surgery. There were no statistical differences in knee circumference, knee range of motion, the day of achieved active straight leg raise, length of hospital stay, the day of knee flexion angle achieved 70 degrees, quality of life, the IOWA help level score, IOWA walking speed score, 10 MWT score and TUG test score between groups after TKR $(p>0.05)$.

Conclusions: In patients with TKR, application of repetitive AROM has better results than CPM to decrease pain severity, improve the HSS knee score. However, AROM has no effect on the early functional outcomes and the other early patients' outcomes. The current results suggest that application of AROM might be an effective method to decrease pain severity, improve the HSS knee score.

References:

[1] Maniar RN, Baviskar JV, Singhi T, et al. To use or not to use continuous passive motion post-total knee arthroplasty: Presenting functional assessment results in early recovery. The Journal of Arthroplasty. 2012;27(2),193-200.

[2] Bruun-Olsen V, Heiberg KE, Mengshoel AM. Continuous passive motion as an adjunct to active exercises in early rehabilitation following total knee arthroplasty-a randomized controlled trial. Disability and Rehabilitation. 2009;31(4), 277-283.

Disclosure of Interest: None declared

DOI: 10.1136/annrheumdis-2017-eular.5851

\section{AB0807 VALIDITY OF THE QUADRICEPS ANGLE MEASUREMENT IN PATIENTS WITH VARUS KNEE OSTEOARTHRITIS: COMPARE THE GONIOMETRIC AND PHOTOGRAMMETRY METHOD TO RADIOGRAPHY METHOD}

M. Erduran ${ }^{1}$, M. Eymir ${ }^{2}$, B. Ünver ${ }^{2}$, N.D. Demirkıran ${ }^{3} \cdot{ }^{1}$ Faculty of Medicine Surgery Medicine Department of Orthopaedics and Traumatology, Dokuz Eylül University, Izmir, Turkey; ${ }^{2}$ School of Physical Therapy and Rehabilitation, Dokuz Eylül University, Izmir, Turkey; ${ }^{3}$ Department of Orthopaedics and Traumatology, Dokuz Eylül University, Izmir, Turkey, Izmir, Turkey

Background: Various measurement methods have been defined to assess the alignment of the lower extremities. The $\mathrm{Q}$-angle measurement is reported to be associated with knee injury and patellofemoral dysfunction. The Q-angle has been assessed using radiography scans, goniometer and photogrammetry methods in supine and standing positions. The radiography method is accepted to be the most accurate and valid measurement technique in measurement the $\mathrm{Q}$-angle. But as a result of being expensive and time-consuming, this invasive method is not mostly preferred in research and clinics. The goniometric measurement of $\mathrm{Q}$-angle is practical because it is simple, requires inexpensive equipment (only a goniometer), and can be applied in a short time. Photogrammetry measurement technique is also an advantageous method for reducing measurement errors caused by goniometers and reduction of investigator errors.

Objectives: The aim of the present study, was to compare the clinical assessment of the Q-angle (goniometric and photogrammetric measurement methods) to a radiological assessment using radiograph (criterion validity) in patients with varus knee osteoarthritis.

Methods: The study group consisted of 15 (median age $68.6 \pm 11.9$ years) patients with unilateral and bilateral varus knee osteoarthritis. Q-angle measurements were assessed on both lower extremities with three different goniometric methods (goniometric measurement method in standing position with quadriceps muscle relaxed; goniometric measurement method in supine position with quadriceps muscle relaxed; and contracted) using a $360^{\circ}$ universal goniometer. After the goniometric measurements, photographs were taken with quadriceps muscle relaxed in standing position. For radiographic assessment, we used patients' lower extremity scanograms, which had been taken to determine the diagnosis and treatment from the Computer Patient Archiving System database.

Results: There was a good correlation between the radiographic assessment and photogrammetric measurement $(r=0.623, p<0.001)$. Also a good correlation was observed between radiographic assessment and goniometric measurement (in standing position with quadriceps muscle relaxed; in supine position with quadriceps muscle relaxed; and contracted) $(r=0.676, p<0.001, r=0.616, p<0.001$, $r=0.676, p<0.001$, respectively).

Conclusions: According to our result, the photogrammetric measurement and the goniometric measurement appear to be valid alternatives to the radiographic measurement for determining the $\mathrm{Q}$-angle. These alternative measures might be used by clinicians and researchers to measure the Q-angle of patients with varus knee osteoarthritis in orthopaedic clinics.

Disclosure of Interest: None declared

DOI: 10.1136/annrheumdis-2017-eular.6827

\section{AB0808 COMPARING THE EFFECTS OF PHYSICAL THERAPY AND NON-STEROIDAL ANTI-INFLAMMATORY TREATMENT ON SLEEP QUALITY, QUALITY OF LIFE AND CLINICAL STATUS IN KNEE OSTEOARTHRITIS}

M. Sezgin ${ }^{1,1}$, E. Yeşildal ${ }^{1}$, H. Ankaralı ${ }^{2}$, G. Sahin ${ }^{1} .{ }^{1}$ Department of Physical Medicine and Rehabilitation, Mersin University Medical Faculty, Mersin;

${ }^{2}$ Department of Biostatistics and Medical Informatics, Duzce University Medical Faculty, Düzce, Turkey

Objectives: We aimed to compare the effectiveness of physical therapy (PT) and non-steroidal anti-inflammatory drug (NSAID) therapy on sleep quality, quality of life and clinical status in patients with knee osteoarthritis (OA).

Methods: Demographic characteristics of the participants were recorded. MOS sleep scale, Nottingham Health Profile (NHP) and Western Ontario ve McMaster Universities Osteoarthritis Index (WOMAC) were used to evaluate sleep quality, quality of life and clinical status of patients before and after the treatment.All patients were divided into two groups as PT (57 patients) and NSAID (43) groups. The patients in the drug group were administered oral dexketoprofen $(50 \mathrm{mg} /$ day, for two week) and the patients in the PT group were administered five times a week, total 15 seances hot-pack, ultrasound and transcutaneous electrical nerve stimulation therapy.

Results: Demographic characteristics, MOS sleep scale and NHP were similar at baseline in both groups $(p>0.05)$. WOMAC pain, stiffness, and physical function scores before treatment were significantly poorer in the PT group than the NSAID group $(P<0.05)$. There were statistically significant improvements on the MOS sleep scale (except snoring subscore, $p=0.05$, table 1 ), NHP (except social isolation, $p=0.209$ ) and and WOMAC pain, stiffness, physical function and total scores of both groups after the treatments $(p<0.05)$. Improvement was also similar when both groups were compared after the treatment $(p>0.05)$. After the two treatment, significantly side effect was not observed.

Conclusions: PT and NSAID therapy has good effectiveness on sleep quality, quality of life and clinical status in knee OA.

References:

[1] Wilcox S, Brenes GA, Levine D, et al. Factors related to sleep disturbance in older adults experiencing knee pain or knee pain with radiographic evidence of knee osteoarthritis. J Am Geriatr Soc 2000; 48:1241.

[2] Vivien CA, Priscilla SA, Christian G. Sleep and rheumatologic disorders. Sleep medicine reviews 2008;12: 211-228.

[3] Dieppe PA, Lohmander LS. Pathogenesis and management of pain in osteoarthritis. Lancet 2005; 365:965-973.54.

[4] Fielden JM, Gander PH, Horne JG, et al. An assessment of sleep disturbance in patients before and after total hip arthroplasty. J Arthroplasty 2003; 18:371-6.

[5] Hunter DJ, Lo GH. The management of osteoarthritis: an overwiev and call to appropriate conservative treatment. Rheum Dis Clin N Am 2008; 34:689-712.99.

[6] Hays RD, Martin SA, Sesti AM, Spritzer KL. Psychometric properties of the Medical Outcomes Study Sleep measure. Sleep Med 2005; 6:41-44.

Disclosure of Interest: None declared

DOI: 10.1136/annrheumdis-2017-eular.3910

\section{AB0809 FALL RISK AND RELATED FACTORS IN KNEE OSTEOARTHRITIS}

M. Alkan Melikoglu ${ }^{1}$, A. Kul ${ }^{2} .{ }^{1}$ Rheumatology; ${ }^{2}$ Physical Medicine and Rehabilitation, Ataturk University Faculty of Medicine, Erzurum, Turkey

Background: Balance, as a complex task, may be affected in knee osteoarthritis $(\mathrm{KOA})$ and this may cause postural instability and fall risk.

Objectives: The aim of this study was to determine the fall risk in patients with KOA with an objective computerized technique and to evaluate the potential risk factors for falls in these patients.

Methods: Patients with KOA and controls were included in this cross-sectional study. Gender, age, and body mass index (BMI) were recorded. Pain was evaluated with a visual analog scale (VAS). The Western Ontario and McMaster Universities Osteoarthritis Index (WOMAC) was used to assess the patients and the Falls Efficacy Scale International (FES-I) was used for the evaluation of fall efficacy. Knee radiographs were recorded with the Kellgren-Lawrence grading scale. Fall risk analysis was performed by using the Tetrax Interactive Balance System, which is a computerized posturography device.

Results: One hundred patients with KOA and 30 controls were included. The age, gender, and BMI scores were similar between the groups. FES-I scores were significantly higher in the cases than in the controls $(p<0.000)$. Using a computerized system, significantly higher fall risk results $(p<0.000)$ and significantly low, moderate, and high fall risk distribution were recorded in the cases than in the controls $(p<0.000)$. Fall risk was significantly related to age, pain, and the WOMAC scores of the patients. 